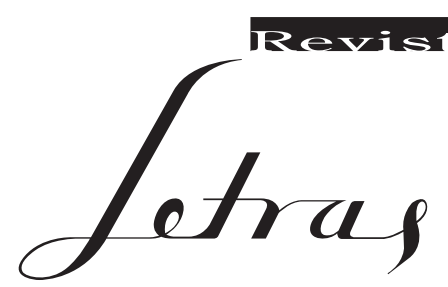

Revista Letras

No 96 - Jul./Dez. 2017

http://revistas.ufpr.br/letras

Editor: Alexandre Nodari

Secretaria Editorial: Rodrigo Otávio Lunardon

Editora da Seção de Estudos Linguísticos: Patrícia de Araujo Rodrigues

Editores da Seção de Estudos Literários:

Mauricio Mendonça Cardozo e Guilherme Gontijo Flores

Diagramação: Yuri Kulisky

Projeto Gráfico: Yuri Kulisky

\title{
Revisão de Textos
}

Beatriz Pires Santana, Camila Moreira, Denise Mazocco, Fernanda Felix, Giselle Ludka, Karina Fernandes, Kayron Beviláqua, Luana de Conto, Maísa Cardoso, Patrícia Sobczynski Gonçalves, Shehrazad Elis Ramos Daoud, Patrícia Rodrigues

\section{Organizadores do dossiê sobre o Workshop on Formal Linguistics}

Roberta Pires de Oliveira, Ana Paula Quadros Gomes, Maria José Foltran e Patrícia Rodrigues

\section{Conselho Editorial}

Antonio Dimas (UsP), Beatriz Gabbiani (Universidad de la República do Uruguai), Carlos Alberto Faraco (UFPR), Carlos Costa Assunção (Universidade de Trás-os-Montes e Alto Douro), Elena Godoi (UfPr), Filomena Yoshie Hirata (USP), Gilda Santos (UfRJ), José Borges Neto (UFPR), Júlio Cesar Valladão Diniz (PUC-RJ), Lígia Negri (UFPR), Lúcia Sá (Manchester University), Lucia Sgobaro Zanette (UFPR), Maria Lucia de Barros Camargo (UfSC), Marília dos Santos Lima (Unisinos), Mauri Furlan (UfSC), Mauricio Mendonça Cardozo (UfPr), Raquel Salek Fiad (UnicAmp), Rodolfo A. Franconi (Darthmouth College), Rodolfo Ilari (UNICAMP)

\section{Conselho Consultivo}

Adalberto Müller (Uff), Álvaro Faleiros (USP), Brunno Vinicius Gonçalves Vieira (unesp-Araraquara), Fernando Cabral Martins (Universidade Nova de Lisboa), Helena

Martins (PUC-RIO), Irene Aron (USP), Isabella Tardin Cardoso (UNICAMP), Juliana Perez (usP), Luciana Villas Boas (UfrJ), Márcia Martins (PUC-Rio), Maria Irma Hadler Coudry (Unicamp), Matthew Leigh (University of Oxford), Patrick Farrell (University of California/Davis) 


\section{Lista dos pareceristas ad hoc}

Ubiratã Alves, Aroldo Andrade, Paula Robert Armelin, Indaiá Bassani, Renato Basso, Roberlei Bertucci, José Borges Neto, Dorothy Brito, Rodrigo Bueno, Janayna Carvalho, Danniel Carvalho, Larissa Ciríaco, Lena Dal Pozzo, Bruno Dallari, Gean Damulakis, Marcelo Ferreira, Maria Cristina Figueiredo Silva, Rossana Finau, Simone Floripi, Maria José Foltran, Álvaro Fujihara, Marcelo Giovannetti, Ana Paula Quadros Gomes, Lara González, Simone Guesser, Márcio Guimarães, Maximiliano Guimarães, Andrea Knöpfle, Marina Legroski, Adriana Lessa, Marcus Vinícius Lunguinho, Alessandro Medeiros, Paulo Medeiros Junior, Sergio Menuzzi, Rafael Minussi, Marcelo Modesto, Bruna Costa Moreira, Andrew Nevins, Gustavo Nishida, Gabriel Othero, Luiz Arthur Pagani, Nize Paraguassu, Ana Lúcia Pessotto, Eloísa Pilati, Roberta Pires de Oliveira, Cristina Prim, Jânia Ramos, Núbia Rech, Pablo Nunes Ribeiro, Cilene Rodrigues, Luciana Sanchez-Mendes,

Filomena Sândalo, Ana Paula Scher, Izabel Christine Seara, Adeílson Sedrins, Marcelo Sibaldo, Leonor Simioni, Luisandro Mendes Souza, Luciana Storto, Helena Guerra Vicente, Teresa Wachowicz, Marcos Carreira 


\section{SumÁrio}

7 On the Morphosyntax of Augmentatives in Brazilian Portuguese Paula Roberta Gabbai Armelin

33 A SEMÂNTICA DE Vetores: UMA PROPOSTA DE ANÁLISE PARA OS ADJUNTOS TEMPORAIS

Renato Miguel Basso

João Francisco Bergamini-Perez

54 IMPOSTORES, CORREFERÊNCIA E CONCORDÂNCIA EM PORTUGUÊS BRASILEIRO Danniel da Silva Carvalho

Dorothy Brito

75 DUAS SOLUÇÕES CONVERSACIONAIS PARA A DESCRIÇão DE INFERÊNCIAS PRESSUPOSICIONAIS EM ENUNCIADOS DE SENTENÇAS NEGATIVAS E CONDICIONAIS COM O GATILHO GANHAR

Marcos Goldnadel

105 A Pronúncia das Vogais Médias no Português Europeu e no Português Brasileiro: um estudo diacrônico

Geruza de Souza Graebin

133 Predicados de Gosto Pessoal em Português BRAsileiro: INDIVIDUAL OU STAGE LEVEL PREDICATES?

Marina Nishimoto Marques

Renato Miguel Basso

153 EM FAVOR Do CONTEÚDO SEMÂNTICO DAS RAÍZE

Rafael Dias Minussi

Indaiá de Santana Bassani

175 ANALISANDO A RETOMADA ANAFÓRICA DO OBJETO DIRETO EM PORTUGUÊS FALADO

Gabriel de Ávila Othero

Ana Carolina Spinelli

197 A arquitetura da sentença no Português Brasileiro: Considerações sobre Sujeito e Tópico

Sandra Quarezemin

220 Predicados inacusativos E a modalidade deôntica

Núbia Ferreira Rech

Giuseppe Freitas Varaschin

240 NOMES PRÓPRIOS E ARTIGOS DEFINIDOS NO PORTUGUÊS BRASILEIRO Adeilson Pinheiro Sedrins

257 Estratégias de RePARO aO ATAQUe RAMificado CCV NA AQUisição FONOLÓGICA

Andressa Toni 
289 Dossier The XI Workshop on Formal Linguistics

Ana Paula Quadros Gomes

Maria José Foltran

Roberta Pires de Oliveira

293 MeAsure WORDS AND CLASSIFIERS

Jenny Doetjes

311 Counting In the verbal Domain

Susan Rothstein

336 Vocatives in the Balkans

Virginia Hill

356 BRAZILIAN BARE NOUNS IN COMPARATIVES: EXPERIMENTAL EVIDENCE FOR NONCONTEXTUAL DEPENDENCY

Kayron Beviláqua

Roberta Pires de Oliveira

379 Time-COUNTING EXPRESSIONS AS ASPECTUAL COMPLEX NUMERALS

Anna Maria Di Sciullo

Manuel Español-Echevarría

400 Italian Causatives AND THE GRAmMar of (IN)DireCt CAUSATION

Marta Donazzan

425 Demonstrativos, Determinantes e definitude em Wapichana

Marcelo Giovannetti

Renato Miguel Basso

444 The Formal and the Relevant: modal underspecification and pragmatic DISAMBIGUATION

Monica Monawar

Claudia Strey

463 Whose foot is it? Pronominal Possessives in Pro-drop Languages: An

EXPERIMENTAL STUDY

Cilene Rodrigues

Lena Dal Pozzo

489 O Papel da (Re)Duplicação na Expressão de Pluracionalidade em Libras Luciana Sanches-Mendes

Rimar Ramalho Segala

André Nogueira Xavier

511 UnDERSTANDING THE INTERACTION BETWEEN DEMONSTRATIVE DEIXIS AND DESCRIPTIVE CONTENT

Lovania Roehrig Teixeira

Sergio de Moura Menuzzi

532 EN TORNO A LA HIPÓTESIS DE LA RELEVANCIA SINTÁCTICA DEL SIGNIFICADO TÉLICO Romina Trebisacce

548 A SintaXe E A SEMÂNTICA DE EXPRESSÕeS NUMÉRICAS Do KaRITIANA

Juliana Vignado Nascimento 


\section{Apresentação}

$\mathrm{O}$

presente número da Revista Letras é dedicado aos estudos linguísticos, naugurando para essa área a nova fase de edições temáticas, iniciada em 2016, com a publicação do número dedicado à literatura. Essa edição conpreende os 25 artigos selecionados da chamada para à edičo do núnero "Linguística Formal". São trabalhos inéditos, que cobrem os grandes tópicos dos estudos contemporâneos, na abordagem formal das línguas naturais: fonética/ fonologia, morfologia, sintaxe, semântica, pragmática, aquisição e interfaces. Além disso, os artigos apresentam uma grande diversidade no que diz respeito às línguas investigadas - português, espanhol, italiano, romeno, inglês, finlandês, libras e as línguas indígenas brasileiras Karitiana e Wapichana - e à procedência dos seus autores, filiados a diversas universidades no Brasil e no exterior. Essa diversidade, representativa do trabalho em Linguística Formal desenvolvido atualmente, encontra, nesse número, artigos que contribuem efetivamente com os diferentes tópicos dos estudos sobre as línguas naturais.

O número é composto por duas seções. A primeira delas conta com 12 artigos submetidos de forma independente. A segunda congrega 13 artigos que são resultados de apresentações durante o XI Workshop on Formal Linguistics, realizado em novembro de 2016, em Curitiba, com a organização de Roberta 
Pires de Oliveira, Ana Paula Quadros Gomes, Maria José Foltran e Kayron Bevilácqua.

Abrindo a primeira seção, temos "On the Morphosyntax of Augmentatives in Brazilian Portuguese”, de Paula Roberta Gabbai Armelin(UFJF); seguem-se os artigos: "A semântica de vetores: uma proposta de análise para os adjuntos temporais", de Renato Miguel Basso (UFSCar) e João Francisco Bergamini-Perez (UFSCar); "Impostores, correferência e concordância em português brasileiro", de Danniel da Silva Carvalho (UFBA) e Dorothy Brito (UFRPE/UAST); "Duas soluções conversacionais para a descrição de inferências pressuposicionais em enunciados de sentenças negativas e condicionais com o gatilho ganhar", de Marcos Goldnadel (UFRGS); "A Pronúncia das Vogais Médias no Português Europeu e no Português Brasileiro: um estudo diacrônico”, de Geruza de Souza Graebin (Universidade de Lisboa); "Predicados de gosto pessoal em português brasileiro: individual ou stage level predicates?”, de Marina Nishimoto Marques (UFSCar) e Renato Miguel Basso (UFSCar); "Em favor do conteúdo semântico das raízes”, de Rafael Dias Minussi (UNIFESP) e Indaiá de Santana Bassani (UNIFESP); "Analisando a retomada anafórica do objeto direto em português falado", de Gabriel de Ávila Othero (UFRGS) e Ana Carolina Spinelli (UFRGS); "A arquitetura da sentença no Português Brasileiro: considerações sobre Sujeito e Tópico", de Sandra Quarezemin (UFSC); "Predicados inacusativos e a modalidade deôntica”, de Núbia Ferreira Rech (UFSC) e Giuseppe Freitas Varaschin (UFSC); "Nomes próprios e artigos definidos no português brasileiro", de Adeilson Pinheiro Sedrins (UFRPE/UAST); encerra a seção o artigo "Estratégias de reparo ao ataque ramificado CCV na aquisição fonológica", de Andressa Toni (USP).

A segunda seção se inicia com uma apresentação do dossiê sobre o Workshop on Formal Linguistics, assinada pelas organizadoras do encontro, seguida dos trabalhos que foram apresentados durante o evento e selecionados para esse número. O dossiê recupera a história dos Workshops no Brasil. A participação da comissão organizadora do XI Workshop on Formal Linguistics nesse número foi fundamental para torná-lo, na nossa opinião, uma contribuição importante para os estudos formais na atualidade. Roberta, Ana Paula e Mazé, muito obrigado!

Esta edição não poderia ter sido realizada sem a valiosa contribuição de alguns dos alunos do Curso de Letras e do Programa de Pós-Graduação em Letras da UFPR na revisão dos artigos. Gostaríamos de agradecer especialmente a Beatriz Pires Santana, Camila Moreira, Denise Mazocco, Fernanda Felix, Giselle Ludka, Karina Fernandes, Kayron Beviláqua, Luana de Conto, Maísa Cardoso, Patrícia Sobczynski Gonçalves e Shehrazad Elis Ramos Daoud.

Desejamos a todos uma boa leitura!

Os Editores 